\title{
Synthesis of Dihydrofuranoquinophenoxazine Analogues as New Antitumor Agents
}

\author{
Younghwa Na \\ College of Pharmacy, Catholic University of Daegu, Gyeongsan, Gyeongbuk 712-702, Korea. E-mail:yna7315@cu.ac.kr \\ Received August 23, 2005
}

Key Words : Dihydrofuranoquinophenoxazine, Psorospermin, Antitumor agents

Psorospermin, isolated from African plant Psorospermum febrifugum, exerted good anti-tumor activity against several human and murine cancer cell lines in vitro and in vivo. ${ }^{1}$ The anticancer pharmacological capacity of psorospermin has attracted researchers to study action mechanism of this compound. ${ }^{2,3,6}$ According to the previous work, xanthone moiety in psorospermin intercalates into the DNA base pairs and the epoxide ring interacts with $\mathrm{N}_{7}$-guanine nucleophile. $^{3}$

The fluoroquinobenzoxazine class analogues have been clinically important and widely used as antimicrobial agents. The pharmacological phenomena of these class drugs are known to be originated by DNA gyrase and topoisomerase IV inhibition. ${ }^{4}$ The similarity of mechanistic aspects between bacterial and eukaryotic DNA topoisomerase II evoked researchers to look for the ability of fluoroquinobenzoxazine analogues as possible antitumor agents. ${ }^{5}$ In the series of the compounds synthesized previously, A-62176 5 a showed good pharmacological activity against several cancer cell lines.

In the structural point of view, both psorospermin and A62176 have tricyclic systems, xanthone and phenoxazine. Those are known to intercalate into the DNA base pairs. The binding modes for these compounds implicated that both are competing for direct interaction at the same DNA intercalation sites in the Drosophila topoisomerase II-DNA complex. ${ }^{6}$ Molecular modeling study also confirmed that the superimposition of psorospermin and A-62176 exhibited substantial structural overlap. ${ }^{7}$ Based on the published research and molecular modeling study information for psorospermin and A-62176, Hurley and his coworkers ${ }^{7}$ designed and synthesized new psorospermin/quinobenzoxazine analogues. In the cytotoxicity test, the hybrid compound exhibited excellent antitumor activity against several human cancer cell lines including breast $\left(\mathrm{IC}_{50}: 0.08 \mu \mathrm{M}\right)$, colon $\left(\mathrm{IC}_{50}: 0.09 \mu \mathrm{M}\right)$, and prostate $\left(\mathrm{IC}_{50}: 0.03 \mu \mathrm{M}\right)$. This result suggested that structural combination of two compounds possessing similar biological profile could produce synergistically enhanced pharmacological activity.

As a continuous work for this compound series, we synthesized new hybrid halohydrin compounds $\mathbf{1}$ and $\mathbf{2}$, different at the stereo-centers on the dihydrofuran ring and its side chain (see structure) with reference compound as epimers of psorospermin/quinobenzoxazine hybrid chlorohydrin. Compound $\mathbf{3}$ was chosen from the retro-synthetic analysis as a key intermediate to construct the skeleton of target compounds, hybrid halohydrins-1 and 2. Compound 3 was prepared as an enantiomeric mixture and the stereochemistry was tentatively assigned based on the information from the literature. ${ }^{7,8}$

We have employed known methods ${ }^{7,8 b, 9}$ for the preparation of final compounds-1 and $\mathbf{2}$. First, compound $\mathbf{3}$ was coupled with ethyl 2,3,4,5-tetrafluorobenzoyl acetate in the triethyl orthoformate and acetic anhydride to give compound 5 and then consecutive double cyclization under basic $\left(\mathrm{NaHCO}_{3}\right)$ furnished pentacyclic compound 6. Regioselective nucleophilic substitution of fluoride at $\mathrm{C}-1$ position in compound 6 with optically pure 3(S)-(tert-butoxycarbonylamino)pyrrolidine was achieved under reflux in pyridine solvent to provide compound $\mathbf{7}$ as a diastereomeric mixture in $60 \%$ yield. We have not tried to separate these two diastereomers. Further elaboration of hydrolysis and followed by selective mesylation at the primary alcohol under pyridine generated key intermediate 9 in $70 \%$ yield after 2 steps (Scheme 1).

Treatment of compound 9 with $\mathrm{K}_{2} \mathrm{CO}_{3}$ in acetone afforded epoxide 10 in moderate yield. In the ${ }^{1} \mathrm{H}-\mathrm{NMR}$ spectroscopy, two doublets appeared at $\delta 2.79(J=4.5 \mathrm{~Hz})$ and $3.32(J=$ $4.5 \mathrm{~Hz}$ ) corresponding to the methylene protons in the epoxide ring. Final halohydrin compounds $\mathbf{1}$ and $\mathbf{2}$ were obtained as diastereomeric mixture with approximately $1: 1$ ratio of two isomers $\left({ }^{1} \mathrm{H}-\mathrm{NMR}\right)$ after mixing with aqueous 1 $\mathrm{N}-\mathrm{HCl}$ or $45 \%-\mathrm{HBr}$ solution. We also prepared compound 11 in which chlorine (or bromine) group of 1 (or 2) was replaced with mesylate. We expect that, if DNA alkylation of compound $\mathbf{1}$ or $\mathbf{2}$ occurs via chlorine or bromine sub-<smiles>COc1cc2c(c3oc4c(O)cccc4c(=O)c13)CC(C1CO1)O2</smiles>

Psorospermin<smiles>NC1CCN(c2c(F)cc3c(=O)c(C(=O)O)cn4c3c2Oc2ccccc2-4)C1</smiles>

A-62176<smiles>C[C@](O)(CCl)[C@H]1Cc2ccc3c(c2O1)-n1cc(C(=O)O)c(=O)c2cc(F)c(N4CC[C@@H](N)C4)c(c21)O3</smiles>

Psorospermin/Quinobenzoxazine Hybrid Chlorohydrin<smiles>[X]CC(C)(O)[C@H]1Cc2ccc3c(c2O1)-n1cc(C(=O)O)c(=O)c2cc(F)c(N4CC[C@@H](N)C4)c(c21)O3</smiles>

1. $\mathrm{X}=\mathrm{Cl}$

2. $\mathrm{X}=\mathrm{Br}$ 
<smiles>CCOC(=O)CC(=O)c1cc(F)c(F)c(F)c1F</smiles>

3<smiles>CCOC(=O)C1=Cn2c3c4c(ccc3c(=O)c3cc(F)c(N5CCC(NC(=O)OC(C)(C)C)C5)c(c32)O1)C[C@@H]([C@](C)(O)CO)O4</smiles>

+ 2-S, 3-R Diastereomer

7<smiles>CCOC(=O)C(=NCNc1c(O)ccc2c1C([C@@](C)(O)CO)C(C(=O)OCC)C2)C(=O)c1cc(F)c(F)c(F)c1F</smiles>

+ Enantiomer<smiles></smiles>

6

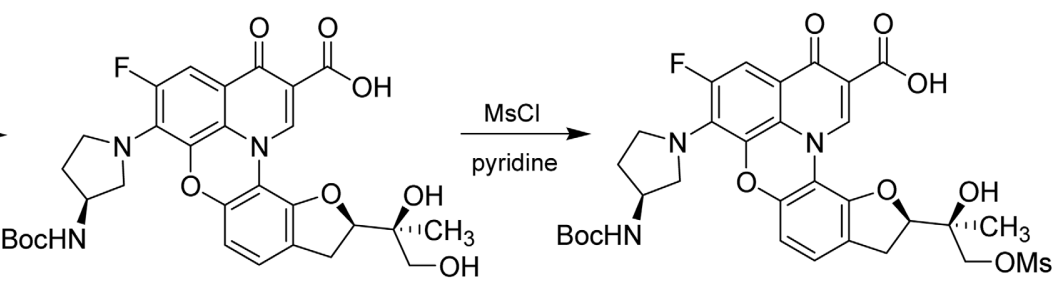

+ 2-S, 3-R Diastereomer

8

9

Scheme 1. Synthetic Method for Key Inermediate Compound 9

stitution pathway, compound $\mathbf{1 1}$ may have similar biological ability.

In conclusion, halohydrin compounds $\mathbf{1}$ and $\mathbf{2}$ were prepared from starting compound $\mathbf{3}$ as epimers of psorospermin/quinobenzoxazine hybrid chlorohydrin in about $6 \%$ total yields. Further cytotoxicity and biological studies are in progress and will be addressed in the future.

\section{Experimental Section}

${ }^{1} \mathrm{H}-\mathrm{NMR}$ spectra were taken on Bruker AMX $300 \mathrm{MHz}$ instrument. Chemical shifts $(\delta)$ are in parts per million (ppm) relative to tetramethylsilane (TMS) as an internal standard and coupling constants ( $J$ value) are in hertz. Low- resolution and high-resolution mass spectral data were recorded at mass spectroscopy center at the University of Arizona. The solvents and reagents were of the best commercial grade available and were used without further purification unless noted.

An enantiomeric mixture of ethyl 3-[2,3-dihydro-2( $R$ )(1,2(S)-dihydroxypropan-2-yl)-6-hydroxybenzofuranyl7-amino]-2-(2,3,4,5-tetrafluorobenzoyl)-2-propenoate and ethyl 3-[2,3-dihydro-2(S)-(1,2(R)-dihydroxypropan-2-yl)6-hydroxybenzofuranyl-7-amino]-2-(2,3,4,5-tetrafluorobenzoyl)-2-propenoate (5): A solution of ethyl 2,3,4,5tetrafluorobenzoyl acetate (4) $(197 \mathrm{mg}, 0.8 \mathrm{mmol})$ in triethyl orthoformate $(0.4 \mathrm{~mL}, 2.4 \mathrm{mmol})$ and acetic anhydride $(0.6$ $\mathrm{mL}, 6.3 \mathrm{mmol})$ was stirred at $130{ }^{\circ} \mathrm{C}(4 \mathrm{~h})$. The solvent was

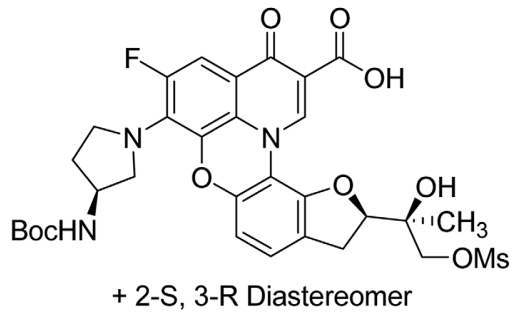

9<smiles></smiles>

+ 2-S, 3-R Diastereomer

10<smiles>COCC(O)(O)[C@H]1Cc2ccc3c(c2O1)-n1cc(C(=O)O)c(=O)c2cc(F)c(N4CC[C@@H](N)C4)c(c21)O3</smiles>

+ 2-S, 3-R Diastereomer

11<smiles>[X]CC(C)(O)[C@H]1Cc2ccc3c(c2O1)-n1cc(C(=O)O)c(=O)c2cc(F)c(N4CC[C@@H](N)C4)c(c21)O3</smiles>

+ 2-S, 3-R Diastereomer

1. $X=\mathrm{Cl}$

2. $\mathrm{X}=\mathrm{Br}$

Scheme 2. Synthesis for Target Compounds $\mathbf{1}$ and $\mathbf{2}$ 
removed in vacuo. The oil residue was dissolved in $\mathrm{CHCl}_{3}$ $(5 \mathrm{~mL})$ and compound $3(150 \mathrm{mg}, 0.7 \mathrm{mmol})$ in $\mathrm{CHCl}_{3} /$ pyridine $(4: 1,2 \mathrm{~mL})$ was added. After stirring at room temperature $(17 \mathrm{~h})$, solvent was removed under reduced pressure. The residue was purified by silica gel column chromatography $\left(13 \% \mathrm{MeOH}: \mathrm{CHCl}_{3}\right)$ to give $174 \mathrm{mg}(52 \%)$ of 5 as yellow solid: $\mathrm{R}_{f}$ : $0.67\left(13 \% \mathrm{MeOH}: \mathrm{CHCl}_{3}\right),{ }^{1} \mathrm{H}-\mathrm{NMR}\left(\mathrm{CDCl}_{3}-\right.$ $\left.\mathrm{CD}_{3} \mathrm{OD}(2: 1), 300 \mathrm{MHz}\right) \delta 1.01(0.94)(\mathrm{t}, J=7.1 \mathrm{~Hz}, 3 \mathrm{H})$, 1.10 (1.08) (s, 3H), 3.01 (2.99) (dd, $J=14.9,9.3 \mathrm{~Hz}, 1 \mathrm{H})$, $3.16(\mathrm{dd}, J=14.9,9.3 \mathrm{~Hz}, 1 \mathrm{H}), 3.61(\mathrm{~m}, 2 \mathrm{H}), 3.99$ (q, $J=7.1$ $\mathrm{Hz}, 2 \mathrm{H}), 4.85$ (dd, $J=9.3,9.3 \mathrm{~Hz}, 1 \mathrm{H}), 6.30$ (6.29) (d, $J=$ $8.0 \mathrm{~Hz}, 1 \mathrm{H}), 6.70$ (6.69) (d, $J=8.0 \mathrm{~Hz}, 1 \mathrm{H}), 9.16(9.15)(\mathrm{s}$, $1 \mathrm{H})$; FAB-MS $m / z 500[\mathrm{M}+\mathrm{H}]^{+}$; FAB-MS $m / z 500.1324$ $[\mathrm{M}+\mathrm{H}]^{+}$(calcd for $\mathrm{C}_{23} \mathrm{H}_{22} \mathrm{NO}_{7} \mathrm{~F}_{4}$ 500.1332).

An enantiomeric mixture of ethyl 1,2-difluoro-4-oxo$4 H$-pyrido[3,2,1-kl]-2(R)-((1,2(S)-dihydroxypropan-2-yl)1,2-dihydrofurano) $[h]$ phenoxazine-5-carboxylate and ethyl 1,2-difluoro-4-oxo-4H-pyrido[3,2,1-kl]-2(S)-((1,2(R)dihydroxypropan-2-yl)-1,2-dihydrofurano) $[h]$ phenoxazine5-carboxylate (6): Compound 5 (144 mg, $0.29 \mathrm{mmol})$ and $\mathrm{NaHCO}_{3}(121 \mathrm{mg}, 1.44 \mathrm{mmol})$ were placed in the DMF (8 $\mathrm{mL}$ ) under nitrogen and the mixture was refluxed at $110^{\circ} \mathrm{C}$ $(2 \mathrm{~h})$. Solvent was removed under reduced pressure and the residue was dissolved in $\mathrm{CHCl}_{3}$ and water. Undissolved material was filtered and organic layer was separated. After removing solvent, the residue was purified by silica gel column chromatography $\left(10 \% \mathrm{MeOH}: \mathrm{CHCl}_{3}\right)$ to give 100 $\mathrm{mg}(75 \%)$ of 6 as weak gray solid: $\mathrm{R}_{f}: 0.52(10 \% \mathrm{MeOH}$ : $\left.\mathrm{CHCl}_{3}\right),{ }^{1} \mathrm{H}-\mathrm{NMR}\left(\mathrm{CDCl}_{3}, 300 \mathrm{MHz}\right) \delta 1.32(\mathrm{~s}, 3 \mathrm{H}), 1.40(\mathrm{t}$, $J=7.1 \mathrm{~Hz}, 3 \mathrm{H}), 3.20$ (dd, $J=15.2,9.5 \mathrm{~Hz}, 1 \mathrm{H}), 3.61(\mathrm{dd}, J=$ $15.2,9.0 \mathrm{~Hz}, 1 \mathrm{H}), 3.69$ (d, $J=11.3 \mathrm{~Hz}, 1 \mathrm{H}), 4.13$ (d, $J=11.3$ $\mathrm{Hz}, 1 \mathrm{H}), 4.34$ (4.28) (q, $J=7.1 \mathrm{~Hz}, 2 \mathrm{H}), 5.00$ (dd, $J=9.5$, $9.0 \mathrm{~Hz}, 1 \mathrm{H}), 6.60(\mathrm{~d}, J=8.1 \mathrm{~Hz}, 1 \mathrm{H}), 7.06(\mathrm{~d}, J=8.1 \mathrm{~Hz}$, 1H), 7.14 (dd, $J=10.1,7.8 \mathrm{~Hz}, 1 \mathrm{H}), 9.62$ (s, 1H).

A diastereomeric mixture of ethyl 1-(3(S)-(tert-butoxycarbonylamino)pyrrolidin-1-yl)-2-fluoro-4-0xo-4Hpyrido[3,2,1-kl]-2(R)-((1,2(S)-dihydroxypropan-2-yl)-1,2dihydrofurano) $[h]$ phenoxazine-5-carboxylate and ethyl 1(3(S)-(tert-butoxycarbonylamino)pyrrolidin-1-yl)-2-fluoro4-oxo-4H-pyrido[3,2,1-kl]-2(S)-((1,2(R)-dihydroxypropan2-yl)-1,2-dihydrofurano) $[h]$ phenoxazine-5-carboxylate (7): Compound 6 (98 mg, $0.22 \mathrm{mmol}$ ) and 3(S)-(tert-butoxycarbonylamino)pyrrolidine (119 $\mathrm{mg}, 0.64 \mathrm{mmol})$ were dissolved in anhydrous pyridine $(6 \mathrm{~mL})$ and the mixture was stirred under nitrogen at $110{ }^{\circ} \mathrm{C}(60 \mathrm{~h})$. The solvent was removed under reduced pressure and the residue was purified by silica gel column chromatography $(10 \% \mathrm{MeOH}$ : $\left.\mathrm{CHCl}_{3}\right)$ to give $80 \mathrm{mg}(60 \%)$ of 7 as yellow solid: $\mathrm{R}_{f}: 0.56$ $\left(10 \% \mathrm{MeOH}: \mathrm{CHCl}_{3}\right),{ }^{1} \mathrm{H}-\mathrm{NMR}\left(\mathrm{CDCl}_{3}, 300 \mathrm{MHz}\right) \delta 1.31$ (1.32) (s, 3H), 1.40 (t, $J=7.1 \mathrm{~Hz}, 3 \mathrm{H}), 1.50$ (1.51) (s, 9H), $1.97(\mathrm{~m}, 1 \mathrm{H}), 2.15(\mathrm{~m}, 1 \mathrm{H}), 3.10(\mathrm{dd}, J=13.5,9.4 \mathrm{~Hz}, 1 \mathrm{H})$, $3.53(\mathrm{~m}, 1 \mathrm{H}), 3.65(\mathrm{~m}, 1 \mathrm{H}), 4.15(\mathrm{~m}, 2 \mathrm{H}), 4.24(\mathrm{~m}, 2 \mathrm{H}), 4.92$ $(\mathrm{m}, 1 \mathrm{H}), 6.29$ (6.32) (d, $J=8.4 \mathrm{~Hz}, 1 \mathrm{H}), 6.91$ (d, $J=8.4 \mathrm{~Hz}$, 1H), $9.26(\mathrm{~s}, 1 \mathrm{H})$.

A diastereomeric mixture of 1-(3(S)-(tert-butoxycarbonylamino)pyrrolidin-1-yl)-2-fluoro-4-oxo-4 $H$-pyrido[3,2,1$k l]-2(R)-((1,2(S)$-dihydroxypropan-2-yl)-1,2-dihydrofurano)- [h]phenoxazine-5-carboxylic acid and 1-(3(S)-(tert-butoxycarbonylamino)pyrrolidin-1-yl)-2-fluoro-4-oxo-4 $\boldsymbol{H}$ pyrido[3,2,1-kl]-2(S)-((1,2(R)-dihydroxypropan-2-yl)-1,2dihydrofurano) $[h]$ phenoxazine-5-carboxylic acid (8): Compound 7 (70 mg, $0.1 \mathrm{mmol})$ was mixed with ethanol $(1.5 \mathrm{~mL})$ and $1 \mathrm{~N}-\mathrm{KOH}(2.0 \mathrm{~mL})$. This mixture was refluxed at $80{ }^{\circ} \mathrm{C}(2 \mathrm{~h})$ and cooled to room temperature. The reaction mixture was acidified with $2 \mathrm{~N}-\mathrm{HCl}(1.0 \mathrm{~mL})$. The solid precipitated was isolated by filtration, washed with water and dried in vacuo to give $60 \mathrm{mg}(90 \%)$ of 8 as orange solid. The product was directly used for next reaction: $\mathrm{R}_{f}: 0.73$ (30\% MeOH : $\left.\mathrm{CHCl}_{3}\right),{ }^{1} \mathrm{H}-\mathrm{NMR}\left(\mathrm{CDCl}_{3}, 300 \mathrm{MHz}\right) \delta 1.25$ (1.24) (s, 3H), $1.51(\mathrm{~s}, 9 \mathrm{H}), 1.98(\mathrm{~m}, 1 \mathrm{H}), 2.17(\mathrm{~m}, 1 \mathrm{H}), 3.10$ (m, 1H), $3.40(\mathrm{dd}, J=14.1,11.1 \mathrm{~Hz}, 1 \mathrm{H}), 3.56(\mathrm{~m}, 1 \mathrm{H}), 3.65$ (m, 1H), $3.79(\mathrm{~m}, 2 \mathrm{H}), 3.93(\mathrm{~m}, 2 \mathrm{H}), 4.32(\mathrm{~m}, 1 \mathrm{H}), 5.00(\mathrm{~m}$, $1 \mathrm{H}), 6.45$ (6.44) (d, $J=8.1 \mathrm{~Hz}, 1 \mathrm{H}), 6.96$ (6.94) (d, $J=8.1$ $\mathrm{Hz}, 1 \mathrm{H}), 7.06$ (d, $J=13.8 \mathrm{~Hz}, 1 \mathrm{H}), 9.54$ (9.52) (s, 1H), 14.49 (s, 1H); FAB-MS m/z $598[\mathrm{M}+\mathrm{H}]^{+}$; FAB-MS m/z 598.2194 $[\mathrm{M}+\mathrm{H}]^{+}$(calcd for $\mathrm{C}_{30} \mathrm{H}_{33} \mathrm{~N}_{3} \mathrm{O}_{9} \mathrm{~F}$ 598.2201).

A diastereomeric mixture of 1-(3(S)-(tert-butoxycarbonylamino)pyrrolidin-1-yl)-2-fluoro-4-oxo-4H-pyrido[3,2,1$k l]-2(R)-((2(S)$-hydroxy-1-methanesulfonyloxypropan-2yl)-1,2-dihydrofurano) $[h]$ phenoxazine-5-carboxylic acid and 1-(3(S)-(tert-butoxycarbonylamino)pyrrolidin-1-yl)2-fluoro-4-oxo-4H-pyrido[3,2,1-kl]-2(S)-((2(R)-hydroxy1-methanesulfonyloxypropan-2-yl)-1,2-dihydrofurano) $[h]$ phenoxazine-5-carboxylic acid (9): To an anhydrous pyridine $(5 \mathrm{~mL})$ solution of compound $8(60 \mathrm{mg}, 0.1 \mathrm{mmol})$ methanesulfonyl chloride $(40 \mathrm{~mL}, 0.51 \mathrm{mmol})$ was added dropwise in the ice-bath. The reaction mixture was stirred at $0{ }^{\circ} \mathrm{C}$ (30 min) and water was added. The solid precipitated was extracted with $\mathrm{CHCl}_{3}$ and the organic layer was washed with water and brine successively. Solvent was removed in vacuo to give $54 \mathrm{mg}(79 \%)$ of 9 as solid: $\mathrm{R}_{f}$ : $0.60(10 \%$ $\left.\mathrm{MeOH}: \mathrm{CHCl}_{3}\right),{ }^{1} \mathrm{H}-\mathrm{NMR}\left(\mathrm{CDCl}_{3}, 300 \mathrm{MHz}\right) \delta 1.38(\mathrm{~s}$, $3 \mathrm{H}), 1.51(\mathrm{~s}, 9 \mathrm{H}), 1.94(\mathrm{~m}, 1 \mathrm{H}), 2.21(\mathrm{~m}, 1 \mathrm{H}), 3.13(\mathrm{~m}, 1 \mathrm{H})$, $3.21(3.20)(\mathrm{s}, 3 \mathrm{H}), 3.53(\mathrm{~m}, 1 \mathrm{H}), 3.55(\mathrm{~m}, 1 \mathrm{H}), 3.73(\mathrm{~m}$, 1H), 3.86 (m, 2H), $4.30(\mathrm{~m}, 1 \mathrm{H}), 4.44$ (4.43) (dd, $J=9.6,2.1$ $\mathrm{Hz}, 1 \mathrm{H}), 4.55$ (dd, $J=9.9,4.8 \mathrm{~Hz}, 1 \mathrm{H}), 5.03$ (dd, $J=9.6,9.6$ $\mathrm{Hz}, 1 \mathrm{H}), 5.15(\mathrm{~d}, J=7.2 \mathrm{~Hz}, 1 \mathrm{H}), 6.49(\mathrm{~d}, J=8.1 \mathrm{~Hz}, 1 \mathrm{H})$, $7.04(7.03)(\mathrm{d}, J=14.4 \mathrm{~Hz}, 1 \mathrm{H}), 7.05(\mathrm{~d}, J=8.1 \mathrm{~Hz}, 1 \mathrm{H})$, $9.51(9.48)(\mathrm{s}, 1 \mathrm{H})$; FAB-MS $m / z 676[\mathrm{M}+\mathrm{H}]^{+}$; FAB-MS $m / z$ $676.1989[\mathrm{M}+\mathrm{H}]^{+}$(calcd for $\mathrm{C}_{31} \mathrm{H}_{35} \mathrm{~N}_{3} \mathrm{O}_{11} \mathrm{FS}$ 676.1976).

A diastereomeric mixture of 1-(3(S)-tert-butoxycarbonylaminopyrrolidin-1-yl)-2-fluoro-4-oxo-4 $H$-pyrido $[3,2,1-k l]-$ 2(R)-(2(S)-methyloxyran-2-yl)-1,2-dihydrofurano[h]phenoxazine-5-carboxylic acid and 1-(3(S)-tert-butoxycarbonylaminopyrrolidin-1-yl)-2-fluoro-4-oxo-4 $H$-pyrido[3,2,1$k l]-2(S)-(2(R)-m e t h y l o x y r a n-2-y l))-1,2-d i h y d r o f u r a n o[h]-$ phenoxazine-5-carboxylic acid (10): To an acetone $(5 \mathrm{~mL})$ solution of compound 9 (54 mg, $0.08 \mathrm{mmol}), 18$-crown-6 $(21 \mathrm{mg}, 0.09 \mathrm{mmol})$ and $\mathrm{K}_{2} \mathrm{CO}_{3}(111 \mathrm{mg}, 0.8 \mathrm{mmol})$ were added. The reaction mixture was stirred $(14 \mathrm{~h})$ and extracted with $\mathrm{CHCl}_{3}$. Organic layer was washed with dil- $\mathrm{HCl}$ and sat$\mathrm{NaCl}$ aqueous solution, successively. After removing solvent under reduced pressure, the residue was purified by PTLC (8\% MeOH : $\mathrm{CHCl}_{3}$ ) to afford $20 \mathrm{mg}$ (43\%) of 10 as 
yellow solid: $\mathrm{R}_{f}$ : $0.64\left(8 \% \mathrm{MeOH}: \mathrm{CHCl}_{3}\right),{ }^{1} \mathrm{H}-\mathrm{NMR}$ $\left(\mathrm{CDCl}_{3}, 300 \mathrm{MHz}\right) \delta 1.49(\mathrm{~s}, 12 \mathrm{H}), 1.97(\mathrm{~m}, 1 \mathrm{H}), 2.20(\mathrm{~m}$, $1 \mathrm{H}), 2.79(2.78)(\mathrm{d}, J=4.5 \mathrm{~Hz}, 1 \mathrm{H}), 3.26(\mathrm{~m}, 1 \mathrm{H}), 3.32(\mathrm{~d}, J$ $=4.5 \mathrm{~Hz}, 1 \mathrm{H}), 3.37(\mathrm{~m}, 1 \mathrm{H}), 3.55(\mathrm{~m}, 1 \mathrm{H}), 3.68(\mathrm{~m}, 1 \mathrm{H})$, $3.74(\mathrm{~m}, 1 \mathrm{H}), 3.93(\mathrm{~m}, 1 \mathrm{H}), 4.34(\mathrm{~m}, 1 \mathrm{H}), 5.07(\mathrm{~m}, 1 \mathrm{H}), 5.24$ $(\mathrm{dd}, J=9.3,8.4 \mathrm{~Hz}, 1 \mathrm{H}), 6.56(\mathrm{~d}, J=8.1 \mathrm{~Hz}, 1 \mathrm{H}), 7.01(\mathrm{~d}, J$ $=8.1 \mathrm{~Hz}, 1 \mathrm{H}), 7.51(\mathrm{dd}, J=13.5,4.8 \mathrm{~Hz}, 1 \mathrm{H}), 9.76(9.72)(\mathrm{s}$, $1 \mathrm{H}), 14.64(14.63)(\mathrm{s}, 1 \mathrm{H})$; FAB-MS $m / z 580[\mathrm{M}+\mathrm{H}]^{+}$; FABMS $m / z 580.2104[\mathrm{M}+\mathrm{H}]^{+}$(calcd for $\mathrm{C}_{30} \mathrm{H}_{31} \mathrm{~N}_{3} \mathrm{O}_{8} \mathrm{~F} 580.2095$ ).

A diastereomeric mixture of 1-(3(S)-aminopyrrolidin1-yl)-2-fluoro-4-oxo-4H-pyrido[3,2,1-kl]-2(R)-((2(S)-hydroxy-1-methanesulfonyloxypropan-2-yl)-1,2-dihydrofurano)[h] phenoxazine-5-carboxylic acid hydrogen chloride and 1-(3(S)-(aminopyrrolidin-1-yl)-2-fluoro-4-oxo-4H-pyrido$[3,2,1-k l]-2(S)-((2(R)$-hydroxy-1-methanesulfonyloxy-propan-2-yl)-1,2-dihydrofurano)[ $h$ ] phenoxazine-5-carboxylic acid hydrogen chloride (11): Compound 9 (9 mg, 0.01 $\mathrm{mmol})$ was dissolved in $1 \mathrm{~N}-\mathrm{HCl}$ aqueous ethyl acetate $(0.8$ $\mathrm{mL})$ and reaction solution was stirred at room temperature $(1$ h). The solvent was removed under reduced pressure and the residue was dissolved in methanol $(3 \mathrm{~mL})$. The solvent was removed. The residue was dissolved in methanol $(3 \mathrm{~mL})$ and $n$-hexane was added into the solution. The solid formed was collected and dried in vacuo to afford $6 \mathrm{mg}(77 \%)$ of $\mathbf{1 1}$ as yellow solid: ${ }^{1} \mathrm{H}-\mathrm{NMR}$ (DMSO- $\left.d_{6}, 300 \mathrm{MHz}\right) \delta 1.21(\mathrm{~s}, 3 \mathrm{H}$ ), $2.03(\mathrm{~m}, 1 \mathrm{H}), 2.27(\mathrm{~m}, 1 \mathrm{H}), 3.24(\mathrm{~m}, 2 \mathrm{H}), 3.26(\mathrm{~s}, 3 \mathrm{H}), 3.71-$ $3.96(\mathrm{~m}, 5 \mathrm{H}), 4.28(\mathrm{~d}, J=9.3 \mathrm{~Hz}, 1 \mathrm{H}), 4.41(\mathrm{~d}, J=9.3 \mathrm{~Hz}$, $1 \mathrm{H}), 5.07$ (dd, $J=9.3,9.3 \mathrm{~Hz}, 1 \mathrm{H}), 5.45$ (br s, $1 \mathrm{H}), 6.80$ $(6.79)(\mathrm{d}, J=8.1 \mathrm{~Hz}, 1 \mathrm{H}), 7.21(\mathrm{~d}, J=8.1 \mathrm{~Hz}, 1 \mathrm{H}), 7.50(\mathrm{~d}, J$ $=14.4 \mathrm{~Hz}, 1 \mathrm{H}), 8.34(\mathrm{~m}, 2 \mathrm{H}), 9.82(9.85)(\mathrm{s}, 1 \mathrm{H}), 14.83(\mathrm{~s}$, 1H); FAB-MS $m / z 576[\mathrm{M}+\mathrm{H}]^{+}$; FAB-MS $\mathrm{m} / \mathrm{z} 576.1455$ $[\mathrm{M}+\mathrm{H}]^{+}$(calcd for $\mathrm{C}_{26} \mathrm{H}_{27} \mathrm{~N}_{3} \mathrm{O}_{9} \mathrm{FS} 576.1452$ ).

A diastereomeric mixture of 1-(3(S)-aminopyrrolidin1-yl)-2-fluoro-4-oxo-4H-pyrido[3,2,1-kl]-2(R)-((2(S)-hydroxy-1-chloropropan-2-yl)-1,2-dihydrofurano) $[h]$ phenoxazine-5-carboxylic acid hydrogen chloride and 1-(3(S)aminopyrrolidin-1-yl)-2-fluoro-4-oxo-4H-pyrido[3,2,1-kl]2(S)-((2(R)-hydroxy-1-chloropropan-2-yl)-1,2-dihydrofurano) $[h]$ phenoxazine-5-carboxylic acid hydrogen chloride (1): Compound $10(7 \mathrm{mg}, 0.01 \mathrm{mmol})$ was dissolved in $1 \mathrm{~N}-\mathrm{HCl}$ aqueous ethyl acetate $(1.0 \mathrm{~mL})$ and reaction solution was stirred at room temperature $(10 \mathrm{~min})$. The solvent was removed under reduced pressure and the residue was dissolved in ether $(5 \mathrm{~mL})$ and sonicated $(2 \mathrm{~min} \times 2)$. The organic layer was decanted and the residue was redissolved in methanol $(3 \mathrm{~mL})$ and concentrated in vacuo to afford 6.5 mg $(90 \%)$ of 1 as orange solid: ${ }^{1} \mathrm{H}-\mathrm{NMR}$ (DMSO- $d_{6}, 300$ MHz) $\delta 1.20(\mathrm{~s}, 3 \mathrm{H}), 2.02(\mathrm{~m}, 1 \mathrm{H}), 2.27(\mathrm{~m}, 1 \mathrm{H}), 3.26(\mathrm{~m}$, $2 \mathrm{H}), 3.69-3.98(\mathrm{~m}, 6 \mathrm{H}), 4.00(\mathrm{~d}, J=10.8 \mathrm{~Hz}, 1 \mathrm{H}), 5.15(\mathrm{dd}$, $J=9.3,8.7 \mathrm{~Hz}, 1 \mathrm{H}), 5.34(\mathrm{~s}, 1 \mathrm{H}), 6.78(6.77)(\mathrm{d}, J=8.1 \mathrm{~Hz}$, $1 \mathrm{H}), 7.20(\mathrm{~d}, J=8.1 \mathrm{~Hz}, 1 \mathrm{H}), 7.53(\mathrm{~d}, J=13.5 \mathrm{~Hz}, 1 \mathrm{H})$, $9.86(\mathrm{~s}, 1 \mathrm{H}) ;$ FAB-MS $\mathrm{m} / \mathrm{z} 516[\mathrm{M}+\mathrm{H}]^{+}$; FAB-MS $\mathrm{m} / \mathrm{z}$ $516.1343[\mathrm{M}+\mathrm{H}]^{+}$(calcd for $\mathrm{C}_{25} \mathrm{H}_{24} \mathrm{~N}_{3} \mathrm{O}_{6} \mathrm{FCl}$ 516.1338).

A diastereomeric mixture of 1-(3(S)-aminopyrrolidin1-yl)-2-fluoro-4-oxo-4H-pyrido[3,2,1-kl]-2(R)-((2(S)-hydroxy-1-bromopropan-2-yl)-1,2-dihydrofurano) [h]phenoxazine-5-carboxylic acid hydrogen bromide and 1-(3(S)-
aminopyrrolidin-1-yl)-2-fluoro-4-oxo-4H-pyrido[3,2,1-kl]2(S)-((2(R)-hydroxy-1-bromopropan-2-yl)-1,2-dihydrofurano) [h] phenoxazine-5-carboxylic acid hydrogen bromide (2): To the $\mathrm{CHCl}_{3}(1 \mathrm{~mL})$ solution of compound $\mathbf{1 0}$ (8 $\mathrm{mg}, 0.01 \mathrm{mmol}$ ) was added 4 drops of $45 \%-\mathrm{HBr}$ aqueous solution. The reaction mixture was stirred at room temperature $(15 \mathrm{~min})$. After removing solvent under reduced pressure, the residue was tritulated in ether $(5 \mathrm{~mL})$ and sonicated $(2 \min \times 2)$. The organic layer was decanted and residue was washed with $\mathrm{CHCl}_{3}(\times 2)$. The residue was redissolved in methanol $(3 \mathrm{~mL})$ and concentrated in vacuo $(\times 2)$. Finally the residue was dissolved in methanol and $n$-hexane was added into the solution. The solid formed was collected and dried in vacuo to afford $8 \mathrm{mg}(91 \%)$ of $\mathbf{2}$ as orange solid: ${ }^{1} \mathrm{H}-$ $\mathrm{NMR}\left(\mathrm{CD}_{3} \mathrm{OD}, 300 \mathrm{MHz}\right) \delta 1.38(\mathrm{~s}, 3 \mathrm{H}), 2.18(\mathrm{~m}, 1 \mathrm{H}), 2.48$ $(\mathrm{m}, 1 \mathrm{H}), 3.18(\mathrm{~m}, 1 \mathrm{H}), 3.32(\mathrm{~m}, 1 \mathrm{H}), 3.66(\mathrm{dd}, J=10.2,4.2$ $\mathrm{Hz}, 1 \mathrm{H}), 3.87(\mathrm{~m}, 3 \mathrm{H}), 4.06(\mathrm{~m}, 2 \mathrm{H}), 5.20(5.18)(\mathrm{dd}, J=9.3$, $9.0 \mathrm{~Hz}, 1 \mathrm{H}), 6.72(6.71)(\mathrm{d}, J=8.1 \mathrm{~Hz}, 1 \mathrm{H}), 7.13(7.12)(\mathrm{d}, J$ $=8.1 \mathrm{~Hz}, 1 \mathrm{H}), 7.27(7.25)(\mathrm{d}, J=13.8 \mathrm{~Hz}, 1 \mathrm{H}), 9.69(9.68)$ (s, 1H); FAB-MS $m / z 560[\mathrm{M}+\mathrm{H}]^{+}$; FAB-MS $m / z 560.0822$ $[\mathrm{M}+\mathrm{H}]^{+}$(calcd for $\mathrm{C}_{25} \mathrm{H}_{24} \mathrm{~N}_{3} \mathrm{O}_{6} \mathrm{FBr} 560.0833$ ).

Acknowledgement. This work was financially supported by Catholic University of Daegu, Korea. The author expresses sincere gratitude to Professor Laurence Hurley at University of Arizona, USA, for his generous help for this work.

\section{References}

1. (a) Kupchan, S. M.; Streelman, D. R.; Sneden, A. T. J. Nat. Prod. 1980, 43, 296-301. (b) Cassady, J. M.; Baird, W. M.; Chang, C.-J. J. Nat. Prod. 1990, 53, 23.

2. Permana, P. A.; Ho, D. K.; Cassady, J. M.; Snapka, R. M. Cancer Res. 1994, 54, 3191.

3. Hansen, M.; Lee, S.-J.; Cassady, J. M.; Hurley, L. H. J. Am. Chem. Soc. 1996, 118, 5553.

4. (a) Bhanot, S. K.; Singh, M.; Chartterjee, N. R. Curr. Pharm. Des. 2001, 7, 313. (b) Anderson, V. E.; Osheroff, N. Curr. Pharm. Des. 2001, 7, 339 .

5. (a) Chu, D. T. W.; Maleczka, Jr. R. E. J. Heterocyclic Chem. 1987, 24, 453. (b) Chu, D. T. W.; Hallas, R.; Clement, J. J.; Alder, J.; McDonald, E. Drugs Exp. Clin. Res. 1992, 18, 275. (c) Chu, D. T. W.; Hallas, R.; Tanaka, S. K.; Alder, J.; Balli, D.; Plattner, J. J. Drugs Exp. Clin. Res. 1994, $20,177$.

6. (a) Kwok, Y.; Zeng, Q.; Hurley, L. H. Proc. Natl. Acad. Sci. U.S.A. 1998, 95, 13531. (b) Kwok, Y.; Zeng, Q.; Hurley, L. H. J. Biol. Chem. 1999, 274, 17226.

7. Kim, M.-Y.; Na, Y.; Vankayalapati, H.; Gleason-Guzman, M.; Hurley, L. H. J. Med. Chem. 2003, 46, 2958.

8. (a) Bigi, F.; Casiraghi, G.; Casnati, G.; Sartori, G. Tetrahedron 1983, 39, 169. (b) Habib, A. M.; Ho, D. K.; Masuda, S.; McCloud, T.; Reddy, K. S.; Aboushoer, M.; McKenzie, A.; Byrn, S. R.; Chang, C.-J.; Cassady, M. J. Org. Chem. 1987, 52, 412. (c) An enatiomeric mixture of 7-Amino-2,3-dihydro-2(R)-(1,2(S)-dihydroxypropan-2-yl)-6-hydroxy-benzofuran (3): ${ }^{1} \mathrm{H}-\mathrm{NMR}\left(\mathrm{CD}_{3} \mathrm{OD}, 300\right.$ MHz) $\delta 1.17(\mathrm{~s}, 3 \mathrm{H}), 3.04(\mathrm{dd}, J=14.3,9.5 \mathrm{~Hz}, 1 \mathrm{H}), 3.17(\mathrm{dd}, J=$ $14.3,9.5 \mathrm{~Hz}, 1 \mathrm{H}), 3.54(\mathrm{~d}, J=10.9 \mathrm{~Hz}, 1 \mathrm{H}), 3.71(\mathrm{~d}, J=10.9 \mathrm{~Hz}$, $1 \mathrm{H}), 4.82(\mathrm{dd}, J=9.5,9.5 \mathrm{~Hz}, 1 \mathrm{H}), 6.26(\mathrm{~d}, J=7.9 \mathrm{~Hz}, 1 \mathrm{H}), 6.45$ (d, $J=7.9 \mathrm{~Hz}, 1 \mathrm{H})$; FAB-MS $\mathrm{m} / \mathrm{z} 225[\mathrm{M}]^{+}$; FAB-MS $\mathrm{m} / \mathrm{z}$ $225.1001[\mathrm{M}]^{+}$(calcd for $\mathrm{C}_{11} \mathrm{H}_{15} \mathrm{NO}_{4} 225.1001$ ).

9. Zeng, Q.; Kwok, Y.; Kerwin, S. M.; Mangold, G.; Hurley, L. H. J. Med. Chem. 1998, 41, 4273. 